\title{
Rapid Resolution of Acute Subdural Hematoma in a Coagulopathic Patient
}

\author{
Navjot Chaudhary, Daria Krivosheya, Elizabeth Small, Cyril Hsia, Wai Ng, Andrew Leung
}

Can J Neurol Sci. 2013; 40: 599-600

Acute subdural hematoma (aSDH) is associated with a 60$80 \%$ mortality rate and is considered a neurosurgical emergency. Although it is most often treated with emergent surgical decompression, patients may be managed conservatively when they are neurologically intact or the hematoma is small. Typical progression of aSDH resolution occurs over weeks, and is characterized by corresponding changes on radiographic imaging where bright aSDH becomes first isointense at about two weeks and then hypointense on noncontrast computed tomogram (CT) head imaging. If there is continued bleeding acutely, however, the SDH may increase in size leading to transtentorial herniation and subsequent clinical deterioration of the patient.

Interestingly, there are a number of case reports in the literature describing the event of spontaneous aSDH resolution. Several hypotheses have been put forward to explain this phenomenon including redistribution of subdural blood and dilution by cerebral spinal fluid $(\mathrm{CSF})^{1}$. Cerebral atrophy, as well as cerebral swelling were both identified to facilitate aSDH resolution ${ }^{2,3}$. Here we describe a patient with an unexpected resolution of an acute SDH in the setting of a bleeding diathesis, and propose that in this patient, coagulopathy contributed to the spontaneous resolution of the hematoma.

\section{Case Presentation}

Our patient is a 73-year-old gentleman who had acute onset of left-sided weakness. His strength was 4-/5 in the left upper and lower extremities. He was alert and oriented, with stable vital signs. His past medical history was significant for myelodysplastic syndrome with chronic pancytopenia, and atrial fibrillation on warfarin therapy.

On admission, significant findings on bloodwork included platelet count of $17 \times 10^{9} / \mathrm{L}$ and an international normalized ratio (INR) of 1.6. Non-contrast CT head scan performed at the initial evaluation demonstrated a moderate-sized acute subdural hemorrhage over the right convexity (Figure 1A). He received vitamin $\mathrm{K}$ and platelets, and was admitted for observation. Five hours later, his level of consciousness decreased, and he required intubation. A follow-up non-contrast CT head demonstrated dramatic enlargement of the acute SDH with uncal herniation (Figure 1B). He received a platelet transfusion and the prothrombin complex concentrate on formulary, Octaplex, in anticipation of emergent surgical evacuation. However, the patient's family instead opted for comfort measures.

Over the next two days, he started to open his eyes and localize to central pain. A follow-up CT performed on Day 2 revealed an unexpected significant resolution of the acute SDH

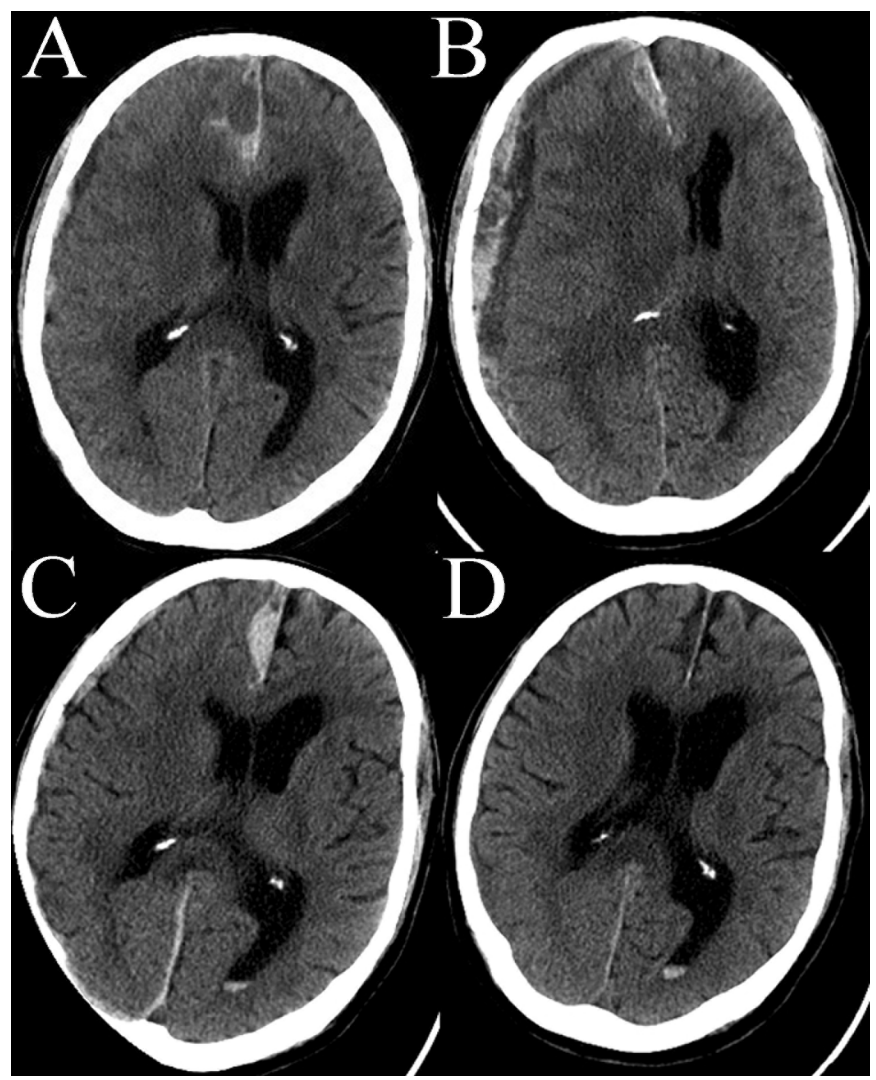

Figure: Non-contrast CT head demonstrating the evolution and the resolution of the acute SDH A. Scan performed on admission demonstrating moderate size aSDH B. Two hours post admission demonstrating significant aSDH expansion causing midline shift. Hypodense component of aSDH likely representing CSF C. Two days post admission, where evidence of blood redistribution is present along falx and tentorium D. Two weeks post admission demonstrating near complete aSDH resolution.

From the Department of Clinical Neurological Sciences (NC, DK, WN), Department of Medical Imaging (ES, AL), Department of Medicine (CH), London Health Sciences Centre, London, Ontario, Canada.

Received August 23, 2012. Final Revisions Submitted January 15, 2013. Correspondence to: Navjot Chaudhary, Department of Neurosurgery, University Hospital, London Health Sciences Center, 339 Windermere Rd, London, Ontario, N6A 5A5, Canada. Email nchaudh3@uwo.ca. 
(Figure 1C). This scan also demonstrated early signs of a right posterior cerebral artery (PCA) infarct in temporal lobe, likely the consequence of PCA compression from uncal herniation. Within several days, he was obeying simple commands and verbalizing. Although he continued to improve radiologically (Figure 1D) and clinically from the neurological standpoint, he unfortunately developed pneumonia and succumbed to this infection 23 days following admission.

\section{Discussion}

Most cases of aSDH are traumatic in origin. As there is an increasing number of patients on oral anticoagulation, however, spontaneous intracranial hemorrhage in a coagulopathic patient in the absence of trauma is becoming more frequent. Irrespective of the etiology, acute symptomatic SDH is a neurosurgical emergency, and majority of patients undergo emergent craniotomy and hematoma evacuation. In some instances, however, there have been documented cases of spontaneous resolution of aSDH. A literature review by Wen et al. in 2009 identified 20 patients who demonstrated the rare phenomenon of spontaneous rapid reversal of acute SDH, typically with resolution or near resolution within 72 hours $^{4}$.

Several mechanisms were put forward to explain rapid resolution of aSDH. The main theory is thought to involve a tear in the arachnoid membrane that may facilitate the influx of CSF into the hematoma resulting in blood clot washout and subsequent redistribution of subdural blood ${ }^{1,4}$. Two factors appear to contribute to the rapid resolution of aSDH: cerebral atrophy by promoting CSF influx ${ }^{2}$ and increased local mass effect secondary to cerebral swelling by facilitating redistribution of blood ${ }^{3}$. In the setting of a head trauma, partial extrusion of the aSDH into the subgaleal space through an overlying scull fracture has been documented to ultimately result in significant reduction of the intracranial component and clinical improvement of the patient ${ }^{5}$.

In the clinical case presented in this paper a number of mechanisms may have contributed to the rapid resolution of the aSDH. The CT head scan performed at the time of clinical deterioration showed an enlarged subdural hematoma consisting of two components: a hyperintense band consistent with continued bleeding, as well as a layering hypointense component consistent with CSF. Hence, it is likely that patient clinical deterioration was at least in part due to CSF trapping within the hematoma cavity. Such a phenomenon has been previously described, resulting in transient patient deterioration ${ }^{1,2,4}$. Moreover, on subsequent imaging there is notable enlargement of the frontal parasaggital aSDH component as well as layering of a hyperintense component consistent with acute blood along the tentorium (Figure 1C) suggesting blood redistribution. Both cerebral atrophy due to the patient's age and cerebral swelling subsequent to the PCA stroke likely promoted aSDH resolution in our patient.
The unique aspect of the case presented above is that the patient had a known double coagulopathy: thrombocytopenia in the setting of myelodysplastic syndrome, and warfarin-induced coagulopathy. To achieve hemostasis, two important components must be intact. Primary hemostasis, or formation of a "platelet plug", requires sufficient quantity of functional platelets working in an intact blood vessel. Secondary hemostasis, or production of a "fibrin clot", requires adequate and functional clotting factors. In this patient, both primary hemostasis, including thrombocytopenia and likely dysfunctional platelets from his myelodysplasia, as well as secondary hemostasis, indicated by the abnormal INR, were involved. While both of these conditions were potential culprits for developing an atraumatic aSDH in our patient, they may have also contributed to the rapid resolution of the hematoma by preventing the formation of a well-organized blood clot and thus be more susceptible to the redistribution via the CSF dilution route. This is the first case report to our knowledge to describe this mechanism of rapid spontaneous aSDH resolution.

\section{SUMMARY}

Spontaneous resolution of aSDH is a rare event that occurs via CSF dilution of the hematoma and subsequent redistribution of blood. Cerebral atrophy and cerebral edema facilitate rapid aSDH resolution. The presented case identifies coagulopathy as a new factor that promotes rapid aSDH resolution.

\section{REFERENCES}

1. Watanabe A, Omata T, Kinouchi H. Rapid reduction of acute subdural hematoma and redistribution of hematoma: case report. Neurol Med Chir (Tokyo). 2010;50(10):924-7.

2. Tsui EY, Fai Ma K, Cheung YK, Chan JH, Yuen MK. Rapid spontaneous resolution and redistribution of acute subdural hematoma in a patient with chronic alcoholism: a case report. Eur J Radiol. 2000;36(1):53-7.

3. Niikawa S, Sugimoto S, Hattori T, et al. Rapid resolution of acute subdural hematoma--report of four cases. Neurol Med Chir (Tokyo). 1989 S;29(9):820-4.

4. Wen L, Liu WG, Ma L, Zhan RY, Li G, Yang XF. Spontaneous rapid resolution of acute subdural hematoma after head trauma: is it truly rare? Case report and relevant review of the literature. Ir J Med Sci. 2009;178(3):367-71.

5. Kundra SN, Kundra R. Extracranial redistribution causing rapid spontaneous resolution of acute subdural hematoma. Neurol India. 2005;53(1):124. 